\title{
Síntomas climatéricos y niveles hormonales
}

\author{
Climateric symptoms and hormonal levels
}

Freeman E, et al. Obstet Gynecol 2007 Aug; 110:230-40.

\section{Objetivo}

Evaluar si los síntomas climatéricos (sofocos, artralgias, síntomas depresivos, trastorno del sueño, disminución de la libido, sequedad vaginal) incrementan su prevalencia durante la transición hacia la menopausia y estimar la asociación de cada uno de ellos con el estadío menopáusico -definido por patrones menstruales- y con los niveles hormonales.

\section{Diseño}

Estudio de cohorte.

Lugar

Pensilvania, EEUU

\section{Pacientes}

Participaron de este estudio 404 mujeres premenopáusicas de 37 a 47 años de edad seguidas durante nueve años en el contexto del Estudio de Envejecimiento del Ovario de Pensilvania. Se realizaron visitas programadas en las que respondieron un cuestionario de síntomas, fechas menstruales y se efectuaron mediciones hormonales de estradiol $\left(E_{2}\right)$, hormona folículo estimulante $(\mathrm{FSH})$ e inhibina $b$ durante la etapa folicular temprana. Las participantes fueron clasificadas como: premenopáusicas (ciclos menstruales regulares de 22 a 35 días), premenopáusicas tardías ( variación en el ciclo en más/menos siete días durante un ciclo), en transición temprana (variación en el ciclo en más/menos siete días durante dos ciclos o dos meses de amenorrea), en transición tardía (tres a 11 meses de amenorrea) o postmenopausias (no histerectomizadas con una amenorrea superior a 12 meses).

\section{Evaluación de factores pronósticos}

Edad, raza, historia de depresión, tabaquismo actual, índice de masa corporal y percepción de estrés.

\section{Medición de resultados principales}

Se estimó la asociación de cada síntoma con el estadío menopaúsico y con los niveles hormonales.

\section{Resultados principales}

El avance del estadio menopáusico se asoció significativamente con un aumento de la prevalencia de sofocos, dolores, artralgias, rigidez y humor depresivo.

Luego de ajustar por el resto de los potenciales confundidores, las mayores fluctuaciones del $E_{2}$ se asociaron con sofocos, dolores, artralgias y envaramiento de las articulaciones; y los niveles mayores de $\mathrm{E}_{2}$ se asociaron con disminución de la libido; mientras que los mayores niveles de $\mathrm{FSH}$, lo hicieron con los sofocos.

No se encontró asociación entre los estadios de la menopausia y la presencia de trastornos en el sueño, disminución de la libido y/o sequedad vaginal.

\section{Conclusiones}

Los estadios en la transición a la menopausia se asocian con sofocos, dolores, artralgias, rigidez y humor depresivo. Las fluctuaciones de los niveles de $E_{2}$ y la disminución de los de inhibina b y el incremento de la FSH se asocian con estos síntomas.

Palabras clave: síntomas climatéricos, niveles hormonales.

Key words: climacteric symptoms, hormonal levels. Fuente de financiamiento: National Institutes of Health.

\section{Comentario}

A veces resulta difícil diferenciar si los síntomas que ocurren durante esta etapa de la vida son debidos a la edad o a la menopausia, como expresión del envejecimiento ovárico.

El estudio de Freeman y col. que comentamos nos muestra que la prevalencia de sofocos, artralgias, dolores, envaramiento y humor depresivo se incrementa durante la transición a la menopausia, con una asociación independiente de la edad y de otros factores de riesgo como el estrés; y que los trastornos del sueño, la sequedad vaginal y la disminución de la libido no se asocian a los estadios de la transición a la menopausia; mientras que las fluctuacines hormonales sí tuvieron asociación a estos síntomas.
Cabe destacar que otras publicaciones ${ }^{1}$ no reportaron similar asociación entre la presencia de síntomas somáticos (artralgia, dolores, rigidez articular) y el estadío menopaúsico; mientras que sí informaron un incremento de la prevalencia de sequedad vaginal con la progresión de la transición a la menopausia.

Si bien el estudio que hemos resumido no aborda el tratamiento de los síntomas climatéricos, podríamos pensar que estabilizando los fluctuantes niveles hormonales característicos de la transición hacia la menopausia lograremos mitigar los síntomas asociados a dicha etapa de la vida.

Marta Tutzer [ Servicio de Ginecología. Hospital Italiano de Buenos Aires. marta.tuttzer@hospitalitaliano.org.ar ]

Tutzer M. Síntomas menopáusicos y fluctuaciones hormonales. Evid. actual. práct. ambul; 11(1):13, Enero-Febrero 2008. Freeman EW et al. Symptoms associated with menopausal transition and reproductive hormones in midlife women. Obstet Gynecol 2007 Aug; 110:230-40. PMID 17666595. Disponible en URL por suscripción: http://www.greenjournal.org/cgi/content/full/110/2/230

\section{Referencias}

1. National Institutes of Health. National Institutes of Health State-of-the-Science Conference statement: Management of menopause-related symptoms. Ann Intern Med 2005; 142:1003-13. 